\title{
La piedra natural en España: evolución y perspectivas
}

\author{
C. Marchán Sanz ${ }^{(1)}$, M. Regueiro y González-Barros ${ }^{(2)}$ y P. Delgado Arenas ${ }^{(2)}$ \\ (1) Ministerio de Industria, Energía y Turismo. Paseo de la Castellana, 160. 28046 Madrid \\ cmarchan@minetur.es \\ (2) Instituto Geológico y Minero de España. Rios Rosas, 23. 28003 Madrid. \\ m.regueiro@igme.es, p.delgado@igme.es
}

\begin{abstract}
RESUMEN
El sector de la Piedra Natural nacional ha sufrido muy duramente el impacto de la crisis del sector de la construcción nacional. La producción tanto en cantera como elaborada se ha resentido gravemente, pasando de 8Mt/año en 2007 a menos de la mitad en 2015, en particular en los subsectores de mármoles y granitos, ya que la pizarra siempre ha sido un subsector netamente exportador. En los últimos tiempos y gracias fundamentalmente a las exportaciones, la producción poco a poco parece que está comenzando a recuperarse. En este trabajo se pasa revista a la tipología de los productos de piedra en España y los datos de producción de los grandes sub-sectores de la piedra natural nacional (mármoles, granitos y pizarras) en los últimos 10 años y se analiza su posible evolución futura en el marco de la incipiente recuperación económica.
\end{abstract}

Palabras clave: España, exportación, importación, piedra natural, producción, tendencias.

\section{Natural Stone in Spain: trends and perspectives}

\begin{abstract}
SUMMARY
The natural stone sector was severely affected by the national construction sector crisis. Production, both exworks and processed, has been also harshly affected dropping from 8Mt/y in 2007 to 3,49Mt/y in 2015, particularly in the marble and granite sectors, since slate has always been a mainly exporting sub-sector. In the latest times, thanks mainly to exports, production apparently has slowly started to recover. In this paper, we review the typology of the Spanish natural stone products and the production data of the main stone subsectors (marble, granite and slate) in the last 10 years and we review possible future trends in the framework of the first steps of the economic recovery.
\end{abstract}

Key words: Spain, exports, imports, natural stone, production, trends.

\section{ABRIDGED ENGLISH VERSION}

\section{Introduction}

Spain has a very varied geology, reflected in the variability of the natural stone typology throughout the country. The natural stone industrial sector has had a long period of expansion and growth from the early nignteens to 2007. Such expansion, parallel to the rapid development of the nation construction sector, halted suddenly in 2008, as the global crisis hit Spain. But during this healthy period, inmense technological advances and upgrading of the national stone manufactuting industry took place, driving production more than $100 \%$ from 1991 to 2007 , thanks to the national market but also to a global expansion internationally.

This paper reviews the geology of the Spanish deposits of natural stone and the economic evolution of the subsector in the framework of the national mining sector, providing insights in the way the sector reacted to the crisis

\section{The natural Stone sector in Spain}

Spain with an overall natural stone production of 3,49 Mt (2015), is currently the $7^{\text {th }}$ stone world producer, after China, India and Iran -who have recently emerged as main global producers-Turkey, Italy and Brazil. This fig- 
Marchán Sanz, C., et al., 2017. La piedra natural en España: evolución y perspectivas. Boletín Geológico y Minero, 128 (2): $395-403$

ure is $50 \%$ less than in 2007 ( $8 \mathrm{Mt}$ ). By sectors marble and limestone marble represented $61 \%$ of the production, granite $19 \%$, roofing slate $19 \%$ and other stones $1 \%$. Roofing slate has suffered less the crisis thanks to the fact that it is mainly an exporting sector. The number of quarries has slightly reduced in the last few years, but the main stone processing centers (around 750) have survived the turmoil of the crisis by reducing production and engaging in an important international trade campaign. Spain exported, in 2013, 1.6 Mt (1.35 Mt in 2014) of marble (more than $76 \%$ of the production) and $70 \%$ of the production of granite. Slate, a traditionally exporting sector, maintained exports in around $80 \%$ of the total production.

\section{Conclusions}

The Spanish stone sector is now facing slightly better perpectives than in the last few years. The domestic market is still flat, but internationally the market is evolving very positively, except in Europe. The fact that the sector has excellent reputation and has the best-known technology used in high quality materials, underpins its potential growing capabilities in the framework of a growing global construction market. But the sector needs also to improve its coordination with other construction sectors to increase the use of stone in buildings, and invest in research and development in fields such as exploration, exploitation design and new technologies, without forgetting the need to adapt their products to an ever evolving and demanding market which asks for new products with new applications and advantages (surface treatments, colors, etc) and all of this done with an extraordinary care for the environment in the framework of sustainable development.

\section{Introducción}

La geología de España es tan compleja y variada que a lo largo de la historia nos ha permitido disponer de muchos materiales útiles que han sido explotados, algunos desde épocas anteriores a los romanos, como prueba la civilización tartesa que hace alrededor de 3000 años habitó la cuenca del Guadalquivir y donde hay constancia de la primera minería organizada en suelo ibérico. Aunque la minería antigua explotaba principalmente yacimientos metálicos, es también bien conocido que los fenicios iniciaron la explotación de sal en lberia, una práctica que continuó ininterrumpidamente durante muchos siglos. En la cartaginense Cartago Nova (actualmente Cartagena, en el SE de España) hubo importantes explotaciones de salmueras marinas para la producción de "garum", una salsa fabricada a base de vísceras de pescado conservado en salmuera (el caviar de aquellos tiempos).

En el caso que nos ocupa, el de las rocas ornamentales, se sabe que los romanos usaron ampliamente el mármol español como roca ornamental para monumentos, no sólo el famoso mármol de Macael (Almería), sino también algún otro afloramiento menos conocido, como uno de cerca de Talavera de la Reina (Toledo) (Urbina et al. 1997).

Después de al menos dos milenios de historia minera, España produjo en 2013 alrededor de 170 millones de toneladas de rocas y minerales industriales (RMI) (una reducción del 70\% desde 2007), con un valor a pie de mina de 1.970 millones de euros (3.800 $M €$ en términos de facturación), lo que supone cerca del $60 \%$ de la producción minera nacional. A pesar de la actual crisis económica y el impacto que está teniendo en la producción y consumo de Rocas y Minerales Industriales (RMI), España sigue siendo un destacado productor a nivel mundial de recursos minerales, en particular en RMI de distintos tipos. Sin embargo la realidad es que la minería apenas representa a pie de mina y en valor absoluto, un escaso 0.3 $\%$ del Producto Interior Bruto nacional.

\section{Panorama de la Minería Nacional 2002-2013}

Los grandes subsectores en que habitualmente se divide la minería, dejando al margen el sector energético, han evolucionado de forma bastante diferente a lo largo del último decenio.

La minería metálica, siempre sujeta a las fluctuaciones de precios, ha pasado de ser casi anecdótica en los primeros años del siglo XXI, con la paralización de todas las explotaciones de la Faja Pirítica y el cierre definitivo de minas tan emblemáticas como Almadén (mercurio, 2001) y Reocín (plomo y cinc, 2003), a tener una fuerte presencia, con importante producción de cobre, níquel, plomo, cinc y valores crecientes de oro, plata, wolframio y estaño. Así, de un valor de producción a pie de mina de $91 \mathrm{M} €$, en 2002, el dato esdtimado para 2015 es de $867 \mathrm{M} €$ (un incremento de casi el $900 \%$ ).

Las rocas y minerales industriales, por su parte, han mantenido cifras crecientes de producción y valor hasta 2007, para iniciarse entonces la crisis generalizada y, con ella, la caída en picado del sector 
de la construcción, lo que ha conllevado el cierre de numerosas explotaciones y reducciones de más del $50 \%$ en las producciones de todas las materias primas relacionadas con la edificación y la obra pública. A pie de cantera, el valor conjunto de la producción de rocas y minerales ha pasado de los $2531 \mathrm{M} €$ en 2002, a los $1994 \mathrm{M} €$ estimados en 2015 (un descenso del $21 \%$ ). De este total, las rocas ornamentales han supuesto en 2015 alrededor de los $387 \mathrm{M} €$

España es el séptimo productor mundial de rocas ornamentales, siendo China e India los mayores productores en la actualidad. Entre otros minerales industriales, España es líder en producción de celestina ( $2^{\circ}$ productor mundial, después de China), sulfato sódico (único productor europeo), fluorita ( $2^{\circ}$ productor europeo), yeso (principal productor europeo y $5^{\circ}$ productor mundial), feldespato (con las mayores reservas de Europa de arenas feldespáticas) sepiolita ( $70 \%$ de las reservas mundiales) y arcillas rojas (destacado productor mundial). Una buena parte de la producción RMI está relacionada con la industria de la construcción.

\section{Geología de la piedra natural en España}

Dada la extraordinaria variedad geológica del país, la distribución de nuestros yacimientos de rocas ornamentales es muy amplia. La Figura 1 muestra la situación de las principales zonas de explotación de piedra natural en España por tipos, sobre un mapa geológico simplificado. Para entender esa ubicación geológi$\mathrm{ca}$, se incluye aquí un breve resumen de la geología de los principales yacimientos de rocas ornamentales españolas (Lombardero et al. 2007).

\section{Granito}

Las canteras de granito se localizan principalmente

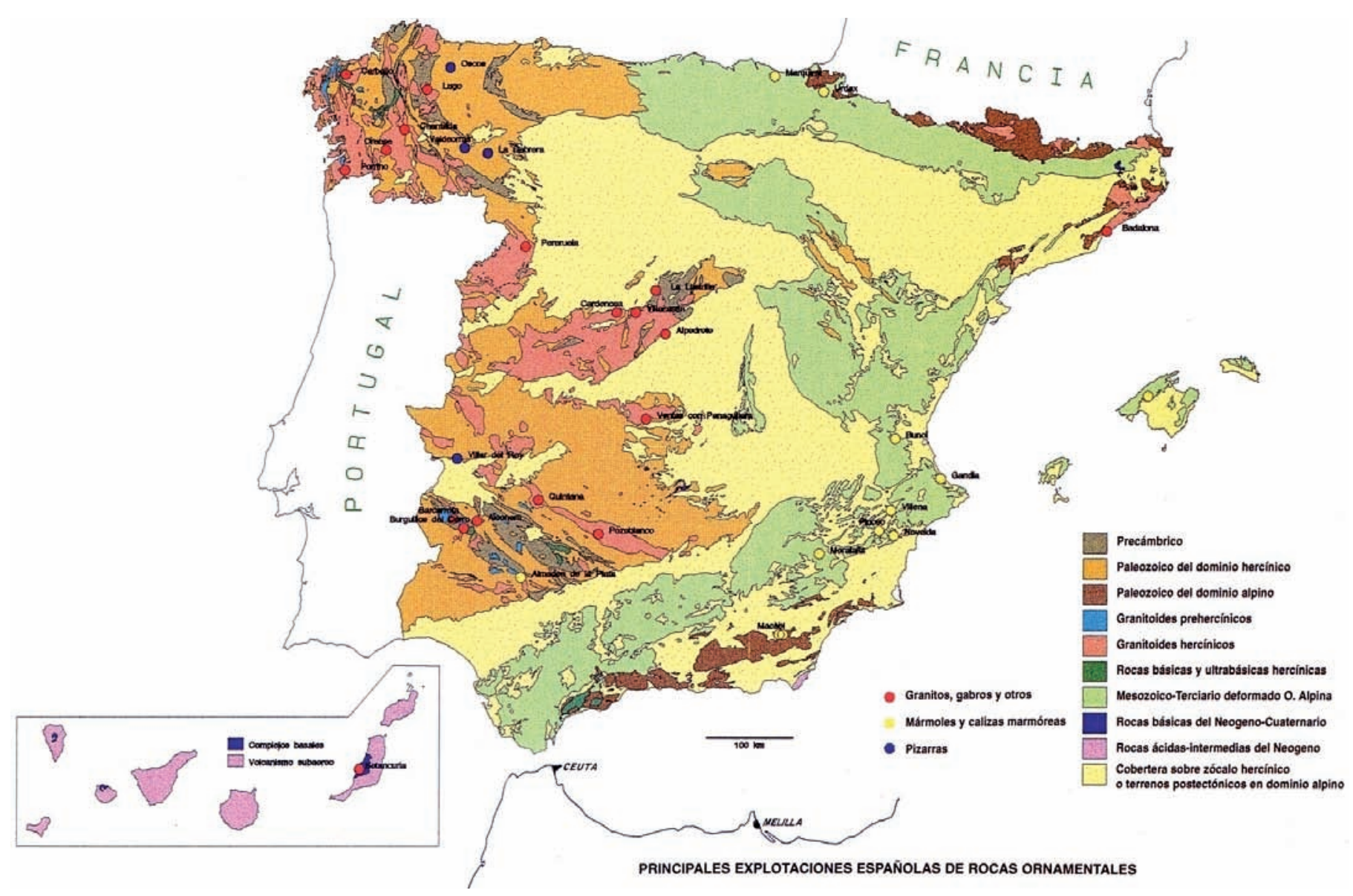

Figura 1. Situación de las principales explotaciones españolas de rocas ornamentales.

Figure 1. Location of the main dimensional Stone operations in Spain. 
en plutones post-variscos. Muchas variedades comerciales pertenecen al tipo "somero no deformado" (Bellido et al. 1987), y son, frecuentemente, granitos rosados, cremas o marronáceos. El color del granito (debido normalmente al color del feldespato y al contenido en biotita) se piensa que es debido a la profundidad de emplazamiento y a la química del cuerpo (por ejemplo, blanco y rosado = somero, gris u oscuro $=$ profundo). Además del basamento varisco, se extraen otros granitos en la zona axial de los Pirineos (incluyendo un extenso dique pegmatítico de alto valor comercial) y en la Cordillera Costero Catalana (Lombardero y Quereda.1992). La composición mineralógica, el tamaño de grano, la presencia de flujo magmático y/o texturas de deformación, primaria (miarolítica) y secundaria (episienitización), porosidad y alteración, son las principales características geológicas que controlan la calidad de un cuerpo plutónico para propósitos ornamentales. Las doleritas, de color verde oscuro, se incluyen también bajo el término comercial "granito".

En Galicia se explotan intensivamente una docena de variedades (incluida la variedad que se exporta a todo el mundo y se conoce como Rosa Porriño). Otras

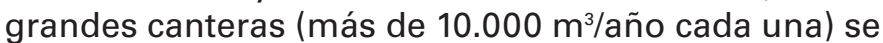
localizan en Extremadura y Madrid (Blanco Cristal, Blanco Aurora, Crema Champán). Pueden encontrarse canteras aisladas en el oeste de Andalucía (Negro Santa Olalla, una tonalita), Salamanca, Zamora, Ávila, Toledo y Lérida (la pegmatita Azul Arán).

\section{Mármol}

El mármol sensu estricto aflora principalmente en la Cordillera Bética, interestratificado con micaesquistos, calcoesquistos y calizas y dolomías recristalizadas. Las capas de mármol están fuertemente plegadas y son muy irregulares, con variaciones en el espesor, número de capas y continuidad lateral. Se explotan en la Sierra de los Filabres (Almería), donde se extrae de muchas canteras pequeñas el famoso Blanco Macael y otras variedades blanca, gris, amariIla y crema. Los carbonatos cámbricos del Macizo Ibérico contienen en muchos lugares mármoles potencialmente valiosos, aunque se explotan en pequeñas canteras (Sierra Alconera en Badajoz, Aroche en Huelva, Guijuelo en Salamanca). La roca ornamental potencialmente más importante de España es la caliza que, aunque no es mármol sensu estricto, se comercializa normalmente bajo esa denominación. Las calizas jurásicas se explotan intensivamente en el sur de España: calizas crema en Granada, Sevilla y Córdoba, calizas rojas en Murcia, Alicante,
Granada y Málaga, y dolomías brechificadas (las valiosas variedades marrones Beige Serpiente y Marrón Imperial) en Valencia. Otras calizas destacables por su valor ornamental son: caliza rosa terciaria (Crema Marfil), caliza marrón y crema del Jurásico Inferior y Cretácico Superior en Valencia y Tarragona, y caliza negra, gris, crema y roja en la cuenca cretácica Vasco-Cantábrica (incluyendo el famoso Negro Marquina). La caliza lacustre horizontal del Plioceno de las cuencas del Duero y Tajo, aunque de pocos metros de espesor, se explota en Valladolid, Burgos y Madrid, gracias a su mínima cobertera, que hace la explotación más fácil, a su calidad de pulido y, algunas veces, a su textura travertino, prescrita por los arquitectos para muchos usos de interior (Lombardero y Quereda. 1992).

\section{Pizarra}

España produce y controla el $80 \%$ del mercado internacional de la pizarra de techar. El valor económico de esta actividad es especialmente importante en el sudeste de Galicia (Valedorras, Orense) y el oeste de León (La Cabrera) donde grandes y pequeñas compañías producen placas de pizarra para techar de alta calidad. La mejor pizarra de techar se explota en formaciones masivas metapelíticas de grado metamórfico inferior a la facies de esquistos verdes (Roberts et al. 1990; García Guinea et al. 1997). Muchas características geológicas afectan a la calidad de la pizarra para techar: leves diferencias en el tamaño de grano y en la composición mineralógica producen cambios destacables en la capacidad de hendido de la roca, los contenidos en pirita y en otros sulfuros de hierro afectan al desarrollo de la pizarrosidad y estructuras menores tales como crenulación, clivaje o kinkbands, el estilo de plegamiento, el grado metamórfico y otros factores, controlan la explotabilidad de los cuerpos de pizarra.

Casi todas las canteras de pizarra de techar de España se localizan en tres formaciones metapelíticas ordovícicas, originalmente depositadas en la plataforma marina de Gondwana. Las pizarras de la Formación Luarca, en la Zona Centroibérica, se caracterizan por ser negras masivas y se explotan intensivamente en los sinclinales de Truchas (Orense y León) y de Alcañices (Zamora). Las formaciones Casayo y Rozadais, del sinclinal de Truchas (Barros 1989) se explotan también intensivamente, aunque se trata de materiales de color gris pálido a oscuro, que afloran en capas de espesores métricos a decimétricos. Capas más finas son sólo explotables en la charnela del pliegue, donde su espesor ha aumentado por ple- 
gamiento similar. También se explota la pizarra negra tremadociense de la Sierra del Caurel (Lugo) y la pizarra verde cámbrica de la formación Pizarras de Cándana, ambas en la Zona Asturoccidental Leonesa. Otras canteras aisladas se localizan en las pizarras devónicas del sinclinal de La Codosera (Badajoz), en la pizarra pre-ordovícica de Bernardos (Segovia) y en la pizarra carbonífera de Sotiel (Huelva).

\section{Piedra Natural en España: Datos económicos}

A pesar de que la producción de piedra natural en España supone un escaso 0,16\% del PIB nacional en 2016 (similar al de 2015), es sector es muy relevante para la economía de las zonas productoras (Comunidad Valenciana, Región de Murcia, Galicia o Castilla y León sobre todo) (Informe Sectorial 2016. Cluster Piedra). Con una producción de alrededor de 3.5 Mt (2013), es actualmente el séptimo productor mundial de rocas ornamentales tras China, India e Irán -que han emergido como principales productores-, Turquía, Italia y Brasil. Estas cifras representan una reducción de más del 50\% respecto al 2007 (8 $\mathrm{Mt}$ ). Por sectores, el mármol y las calizas marmóreas representaron el $58 \%$ de la producción, el granito el $18 \%$, la pizarra el $18 \%$ y las piedras de cantería el $6 \%$. El menos afectado por la crisis fue la pizarra ya que su producto se dirige fundamentalmente a la exportación.

Este sector se encuentra ampliamente distribuido en el territorio nacional, con más de 750 fábricas de elaboración y muchas canteras (563, en 2013). Solamente un $10 \%$ de las empresas llevan a cabo el proceso de producción al completo, desde la extracción en cantera hasta la distribución y venta final. La reducción de la producción ha significado la suspensión de la producción en casi un $70 \%$ de las canteras activas, ya que en 2007 había 916 y en seis años el número de canteras es de 563.

\section{Evolución de la producción en bruto}

Como ya se ha dicho, la producción de rocas ornamentales ha sufrido un fuerte recorte desde el año 2007. La Figura 2 (Estadística Minera de España), refleja la producción de rocas ornamentales nacional en el período 2006-2015 en miles de toneladas, donde se observa el fuerte descenso de la producción sufrida por el sector a partir de 2007. La caída en producción hasta el 2015 ha sido de un 56\%, desde $8 \mathrm{Mt}$ de producción total, a 3,5Mt en 2015. Por sectores y en ese período, la producción de mármol cayó un
GRÁFICO 0.4.1. PRODUCCIÓN DE ROCAS ORNAMENTALES (2008 a 2015). (miles de toneladas)
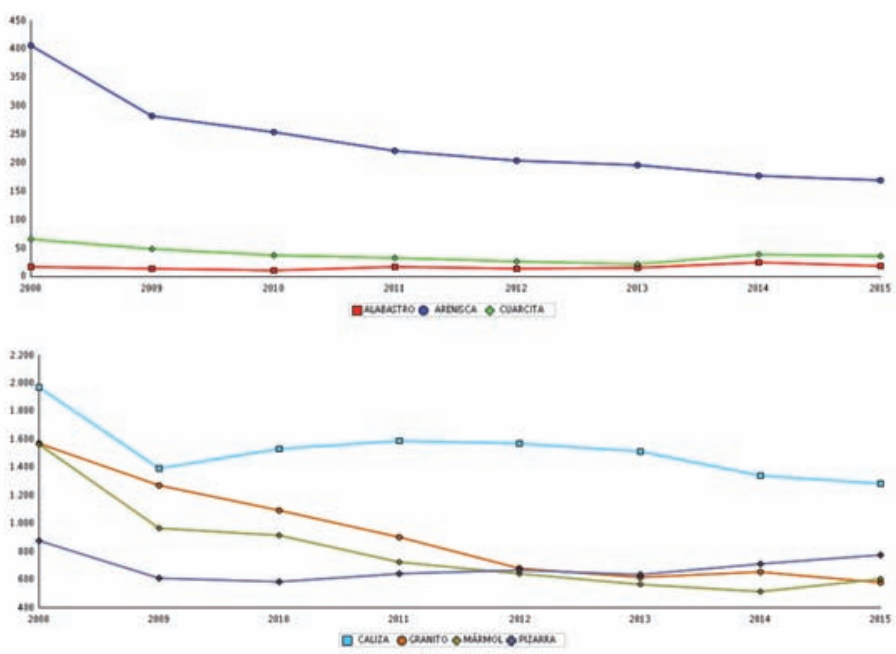

Figura 2. Producción de rocas ornamentales en España 2006-2015 (kt).

Figure 2. Dimensional Stone production of Spain 2005-2015 (kt).

$67 \%$, desde $5.71 \mathrm{Mt}$ a $1.9 \mathrm{Mt}$, la de granito un $62 \%$ desde $1.55 \mathrm{Mt}$ a $0.6 \mathrm{Mt}$, y la pizarra un $17 \%$ desde $0.94 \mathrm{Mt}$ a $0,8 \mathrm{Mt}$.

\section{Producción de producto elaborado}

La tendencia a la reducción de la producción de los últimos años no se ha visto reflejada en el cierre de plantas pero si en un profundo descenso de la producción de baldosas de piedra natural. La figura 4 refleja una estimación del número de plantas de pro-

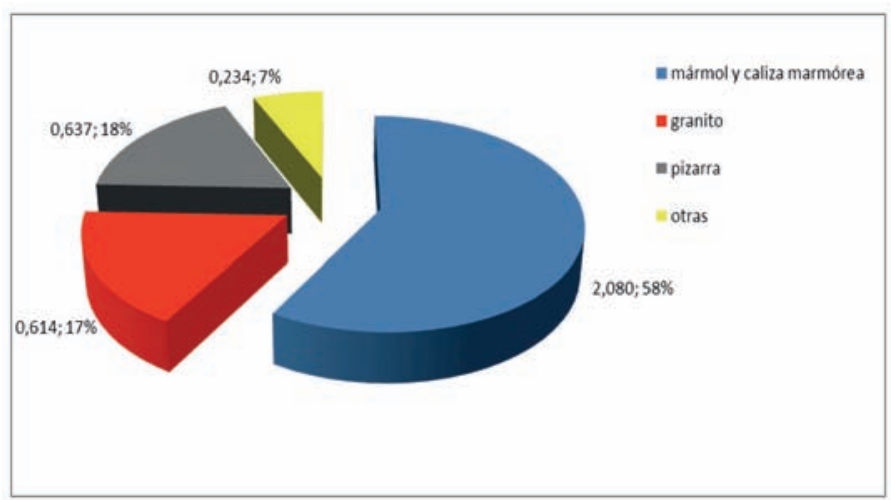

Figura 3. Producción de rocas ornamentales en España en 2013 por tipos de rocas $(\mathrm{Mt})$.

Figure 3. Dimensional stone production pof Spain in 2013 by rock type $(M t)$. 


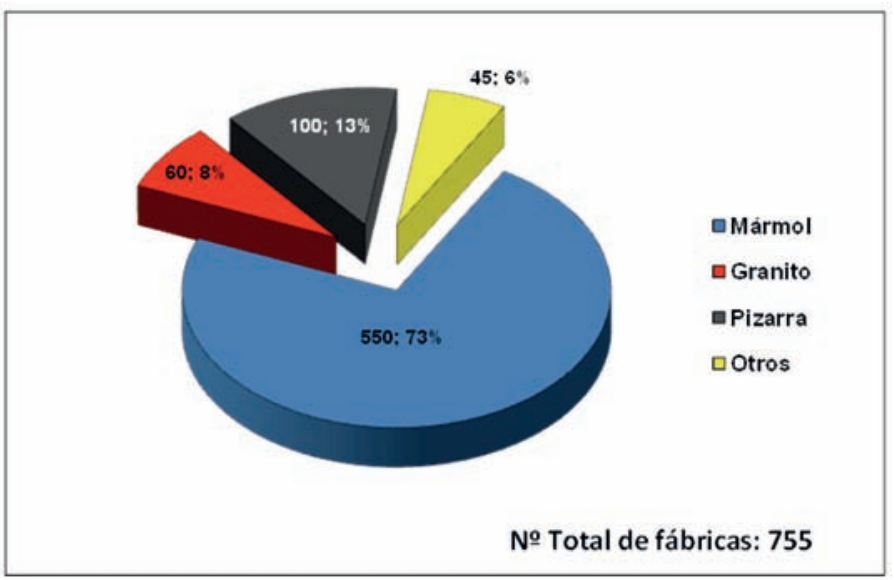

Figura 4. Número de fábricas en España en 2013 por sectores. Figure 4. Natural stone manufacturing plants in Spain (2013).

ducción de elaborados de piedra natural en España en 2013. Desde el año 2009, en que había en España algo más de 800 fábricas, no se han producido cierres de grandes fábricas, solo de un pequeño procentaje de alrededor de un $5 \%$ de fábricas pequeñas y sobre todo del sector del mármol, con lo que la estimación, a falta de datos oficiales contrastados, es de que en 2013 el número de fábricas ronda las 755. No obstante aunque las grandes y medianas empresas no hayan cerrado las plantas de elaboración, para evitar el cierre han acometido un fuerte ajuste de la plantiIla. Según la Federación Española de la Piedra Natural y Afines (Fernández Cortés, F.J. y Caparrós Pérez, D. 2014), el número de empresas que han desaparecido estos años en mucho mayor entre las dedicadas a la explotación debido a que estas empresas suelen tener un mayor volumen de activos que mantener $y$ menor flexibilidad para afrontar los descensos de facturación.

En cuanto a la producción de baldosas, la crisis ha producido un descenso desde 2009 del $42 \%$ en la producción de mármol, un $31 \%$ en la producción de placa de granito y un $2.6 \%$ en el de otras piedras. Por el contrario, la producción de pizarra ha experimentado un ascenso de casi un $24 \%$ debido a su tradicional capacidad exportadora. La producción global de placas de piedra natural fue en 2013 de $70.09 \mathrm{Mm}^{2}$, frente a los $91.35 \mathrm{Mm}^{2}$ de 2009.

\section{El comercio exterior de piedra ornamental}

Frente a la situación claramente deficitaria de otros sectores mineros, como el energético o el de los metales, la roca ornamental siempre ha supuesto un

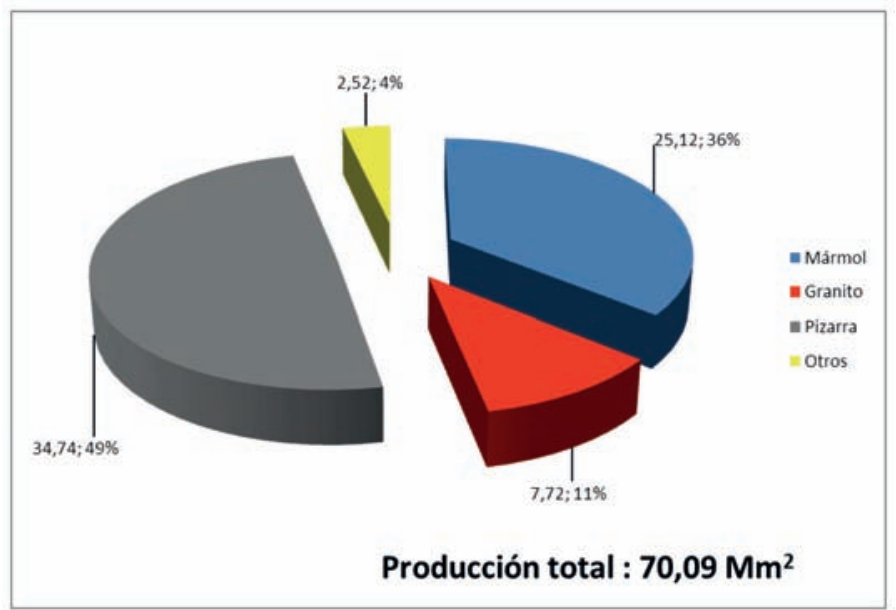

Figura 5. Producción de baldosas de piedra natural en España en 2013 por sectores.

Figure 5. Natural Stone tile production by sectors in 2013.

superávit en la balanza comercial española (Panorama Minero). En los últimos diez años, salvo el mínimo de 2009, con poco más de $500 \mathrm{M} €$ de saldo positivo, el superávit ha rondado o superado los 600 $\mathrm{M} €$ con un máximo cercano a los 780 en 2003, seguido por los $750 \mathrm{M} €$ alcanzados en 2014. Esta buena cifra se ha debido, sin embargo, no tanto al crecimiento de las exportaciones y los precios de las mismas, como al fuerte descenso de las importaciones, debido a la escasa demanda interna.

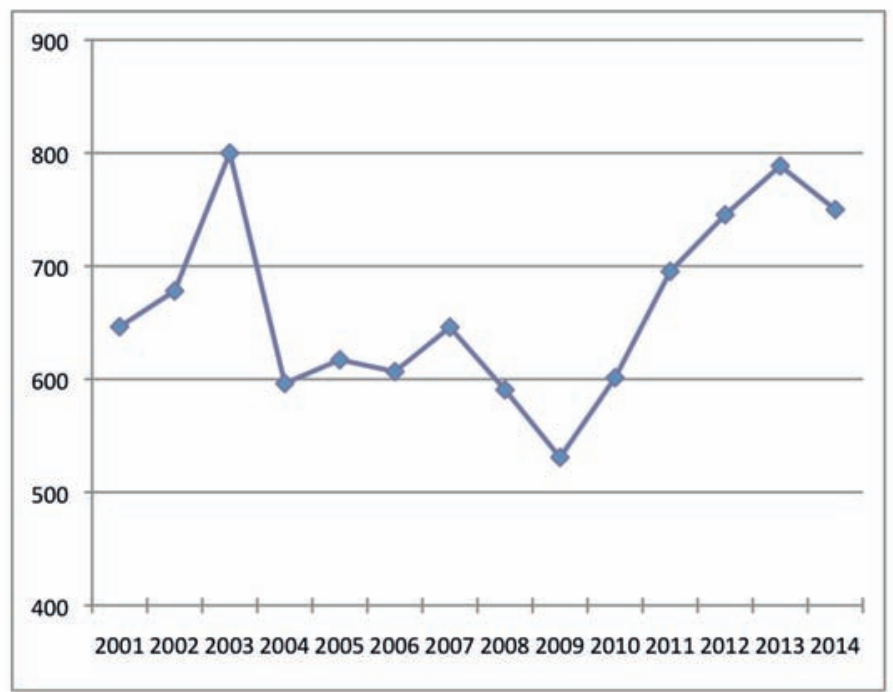

Figura 6. Evolución del saldo del comercio exterior de Piedra Natural 2001-2014 (M€).

Figure 6. Natural Stone Spanish foreign trade 2001-2014 (M€). 
La estructura del comercio exterior es un tanto diferente según se trate de granito o de mármol y pizarra. En el caso del granito, las cifras de importación y exportación son relativamente parecidas, y en muchos ejercicios la importación incluso ha sido superior, en tonelaje, a la exportación. En el caso de mármoles y pizarra, las cantidades importadas son muy inferiores a las exportadas.

También existe otra diferencia importante en cuanto al tipo de producto, ya que las cantidades de granito en bruto importadas suelen ser muy elevadas, en tanto que en el caso de mármol y pizarra son bastante bajas. En cuanto a la exportación, la pizarra en bruto es muy poco significativa, pues apenas alcanza el $2,8 \%$ del total exportado.

El notable descenso de la construcción ha hecho que en los últimos años la demanda interna de rocas ornamentales haya descendido de manera muy acusada, lo que, por otra parte, ha llevado a que las exportaciones se hayan elevado de manera muy importante. Así, por ejemplo, si a comienzos del presente siglo, la cantidad total de mármol exportado se

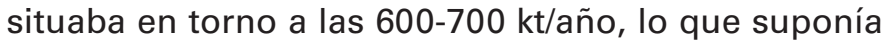
menos del $20 \%$ de la producción total, en 2015 se han exportado 1,23 Mt, casi un $70 \%$ de la producción. Con el granito está ocurriendo algo similar, en 2002 la exportación supuso un $38 \%$ de la producción y en 2015 se estima que ha pasado a ser del $86 \%$. Sin embargo la mayor parte de la piedra que exporta España es en bruto frente al producto elaborado salvo naturalmente en el caso de la pizarra que se trata de pizarras de techar- lo cuál implica una gran pérdida del valor añadido de nuestras exportaciones de piedra natural.

La pizarra ha mantenido un comportamiento más estable a lo largo de estos años ya que siempre ha tenido un elevado potencial exportador, que hace que como mínimo el $80 \%$ de la producción se destine al mercado exterior.

En cuanto al destino de nuestras exportaciones, y ya con datos de 2014, en el caso de la pizarra éste es claramente europeo y no ha variado mucho en la última década: Francia importa el $41 \%$ de nuestra pizarra de techar, Alemania alrededor del 16,6\%, Reino Unido $28 \%$, Bélgica el $6 \%$ y otros países UE el $9 \%$ del total de la exportación.

El granito bruto exportado tiene un claro destino europeo, siendo los principales compradores Portugal (54\%), Italia (14\%), Francia (9\%) y Polonia (8\%). El granito simplemente aserrado se reparte un poco más, aunque también en el entorno próximo, con Francia como principal importador (19\%), seguido de Arabia Saudí (13\%), Marruecos (11\%), Suiza y Portugal (9\%), Argelia y Polonia (5\%). Cuando se trata de granito manufacturado los principales compradores son EEUU (20,5\%), Turquía (13\%), Marruecos $(13 \%)$, Francia (7\%), Portugal y Alemania (5\%), Reino Unido (4\%), Arabia Saudí (3\%) y otros, 80 países (28\%).

El mármol bruto, que en 2002 era exportado en proporciones similares a EEUU (17\%), China (17\%) e Italia (15\%), principalmente, ha pasado en 2015 a tener como claro destino China, que compra el $80,9 \%$ de nuestra producción, Hong Kong, ronda el $3,4 \%$ y Egipto el 2,8\%. El 13\% restante corresponde a 56 países.

El mármol aserrrado se ha dirigido sobre todo a Arabia Saudí (26\%), Emiratos Árabes Unidos (14\%), Oatar $(11 \%)$, Francia $(10,7 \%)$, China $(6,8 \%)$. En cuanto al trabajado, los principales compradores son EEUU (15\%) y Méjico (7\%).

\section{Facturación del sector}

Como era esperable, la caida de la producción ha provocado un drastico descenso de la facturación (Figura 7), desde los valores record de 2008 de $4170 \mathrm{M} €$ a los $1887 \mathrm{M} €$ de 2011 , una caida del $54.7 \%$. Según Cluster Piedra (op cit. 2014), la producción del sector en 2014 alcanzó un valor de $1720 \mathrm{M} €$, un 2,5\% más baja que el año 2013 (1764 M€), lo que haría que la reducción de la facturación desde 2008 alcanzara el 59\% lo que da una idea de la gravedad de la crisis sufrida por el sector en estos años.

\section{Perspectivas de futuro}

El sector español de la piedra natural se encuentra en una encrucijada tras el colapso de la producción experimentado a partir de 2007, pero su futuro plantea muchas posibilidades de mejora, debido a que se dispone de amplios recursos para abastecer el mercado nacional y la red internacional exportadora. Además España tiene aún una extraordinaria capacidad técnica-tecnológica adquirida en la década de crecimiento sostenible previo a la crisis, tanto en producción minera como en el posterior proceso de elaboración (telares, recuperación de residuos, calidad de los materiales obtenidos) y una calidad del producto reconocida internacionalmente $\mathrm{y}$ mantiene intactos sus canales de comercialización. Dentro del país, la red de comunicaciones nacional facilita el acceso de nuestros productos a esos canales.

No obstante lo anterior, el sector tiene algunos claroscuros, como la necesidad de diversificar la oferta de productos en variedad, colores y texturas, con la 


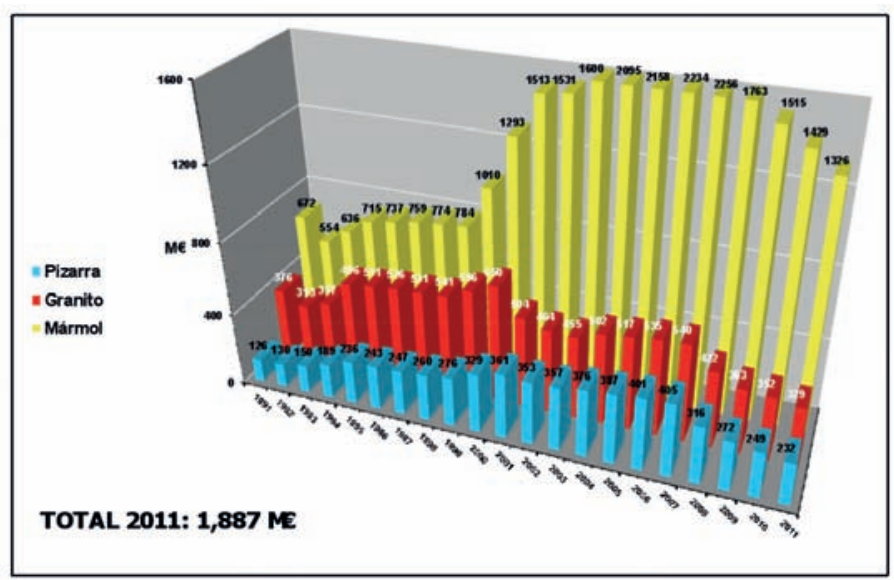

Figura 7. Evolución de la facturación del sector de piedra natural 2002-2011.

Figure 7. Trends of the Spanish natural stone sector turnover 20022011.

apertura de canteras en otros países para cubrir en parte la demanda externa, entre otras cosas porque pese al supéravit de producción nacional, es necesario importar piedra diferente de otros países para cubrir parte de la demanda, es decir, importar más material bruto y menos elaborado, lo que permitiría ganar valor añadido, aprovechando que las instalaciones para elaborarlo las tenemos, tal y como han hecho los italianos tradicionalmente.

Entre los retos que se plantean al sector está la necesidad de una mayor coordinación con el sector del diseño en construcción (arquitectos, constructores, instaladores), para que integren cada vez más la piedra en sus obras, ampliar los mercados, hacia países con potencial de compra que aún no conocen la piedra española (o la importada elaborada en España, que podría comercializarse allí), continuar la investigación en los diferentes productos para los tratamientos y acabados de las diferentes rocas, sistemas de colocación más seguros, etc. La FDP (op cit. 2014) sugiere centrarse en países como China, EEUU, Alemania, Méjico y Rusia.

Finalmente el sector debe reactivar urgentemente sus organizaciones de representación tras la reducción de las actividades de la Federación de la Piedra Natural y Afines (FDP) que ha causado la crisis para retomar con fuerza la presencia en ferias internacionales, el mejor camino para ganar mercados fuera del país.

Pero ante estos retos, el sector empieza a vislumbrar un futuro algo mejor, debido a que la demanda de piedra natural en el mundo sigue al alza, salvo en Europa, donde es previsible que cuando se consolide la recuperación comenzará a crecer. Esas perspecti- vas hacen que la situación financiera de las empresas exportadoras haya mejorado y frente al estado decrépito de la demanda nacional, no queda otra que mejorar posiciones en la arena internacional.

\section{Conclusiones}

España tiene un formidable sector de la piedra natural tanto en variedades producidas como en calidad del producto y sus factorías cuentan con la mejor tecnología disponible, gracias a ello aunque ha sufrido el fuerte impacto de la crisis reduciendo drásticamente su producción, se ha adaptado rápidamente a la situación. No se ha producido el cierre masivo de empresas a pesar de la contracción de la demanda nacional, en gran medida gracias a la proyección internacional del sector productivo. Prueba de ello es que en 2015 se han exportado 1,23 Mt de piedra natural, casi el $70 \%$ de la producción. Por sectores, tanto mármoles como granitos han experimentado profundos descensos en la producción, mientras que la pizarra ha mantenido una posición estable en toda la crisis. Las perspectivas de futuro son favorables para la piedra ornamental, gracias a que a nivel mundial el sector crece en todo el mundo salvo en Europa. Si además el país empezara a crecer, siquiera levemente, la piedra mejoraría su producción con rotundidad.

\section{Referencias}

Barros, J.C. 1989. Nuevos datos geológicos y cartográficos sobre el flanco sur del Sinclinorio deTruchas. Cuadernos del Laboratorio Geológico de Laxe. 14, 93-116.

Bellido, F., González-Lodeiro, F., Klein, E., Martinez-Catalán, J.R. \& Pablo-Macia, J.G. 1987. Las rocas graníticas hercínicas del norte de Galicia y del occidente de Asturias. IGME. Madrid. Memorias, 101.

Fernández Cortés, F.J. y Caparrós Pérez, D. 2014. Plan Estratégico de la Federación Española de la Piedra Natural.

Cluster Piedra. Informe sectorial 2014.

Cluster Piedra. Informe sectorial 2016

Lombardero, M., Quereda, J.M. 1992. La Piedra Natural para la construcción. En: García-Guinea, J., \& Martinez-Frias, J. (Eds) Recursos Minerales de España. Consejo Superior de Investigaciones Cientificas, Colección Textos Universitarios, 15, 1115-1152.

Lombardero, M., Regueiro, M., López-Vera, F., Martinez del Olmo, W., Mallo García, J.M., Saenz de Santamaria, J.A., García Palomero, F., Higueras, P., Ortega, L. 2007. Los recursos geológicos de España. En: Recursos de la Tierra: Origen, uso e impacto ambiental. $3^{a}$ Edición. Craig, J.M., Vaugham, D.J.,Skinner, B.J. pp 557- 609. Pearson Educación. Madrid. 
Trío Maseda, M., Regueiro y González Barros, M. Panorama Minero 2015 (avance). IGME.

Ministerio de Industria, Energía y Turismo. 2013. Estadística Minera de España http://www.minetur.gob.es/energia/mineria/Estadistica/Paginas/Consulta.aspx

Regueiro, M., Marchán, C. y Calvo, J.P. 2010. La industria española de rocas y minerales industriales. Actas del $1^{\text {er }}$ Congreso Nacional de Minerales Industriales. Zaragoza
25-28 mayo 2010. Pp 9-23. Fecha 2010. Ed. Fueyo Editores. Madrid.

Roberts, B., Morrison, C. \& Hirons, S., 1990. Low grade metamorphism of the Manx Group: A comparative study of white mica crystallinity techniques. Journal of the Geological Society of London. 147, 271-277.

Urbina, D., Sánchez, A., Lombardero,M. y Regueiro,M. 1997. Mármoles romanos y canteras en Talavera de la Reina. Zephyrus, 50, 1997, 273-287. Universidad de Salamanca.

Recibido: diciembre 2017

Revisado: febrero 2017

Aceptado: abril 2017

Publicado: junio 2017 
